\title{
8. MATERNAL COUNTRY OF BIRTH
}

In this section maternal countries of birth are combined into English-speaking and other regional groups. The country groups and individual countries are listed in Appendix 3. Recent trends in confinements for individual maternal countries of birth are shown in Table 4 (page 21).

\section{Trends in confinements}

Between 2002 and 2006, about 20 per cent of mothers were born in non-English speaking countries (Table 85). Over the 5 year period, there was a slight decline in the percentage of mothers born in South East Asia, and a slight increase in the percentage of mothers born in South Asian countries.

\begin{tabular}{|c|c|c|c|c|c|c|c|c|c|c|}
\hline \multirow[t]{3}{*}{ Country of birth group } & \multicolumn{10}{|c|}{ Year } \\
\hline & \multicolumn{2}{|c|}{2002} & \multicolumn{2}{|c|}{2003} & \multicolumn{2}{|c|}{2004} & \multicolumn{2}{|c|}{2005} & \multicolumn{2}{|c|}{2006} \\
\hline & No. & $\%$ & No. & $\%$ & No. & $\%$ & No. & $\%$ & No. & $\%$ \\
\hline \multicolumn{11}{|l|}{ Confinements } \\
\hline English-speaking & 67277 & 79.5 & 67322 & 79.2 & 66621 & 79.0 & 70354 & 78.9 & 71515 & 78.3 \\
\hline Central \& South America & 739 & 0.9 & 692 & 0.8 & 675 & 0.8 & 726 & 0.8 & 765 & 0.8 \\
\hline Melanesia, Micronesia \& Polynesia & 1534 & 1.8 & 1545 & 1.8 & 1584 & 1.9 & 1485 & 1.7 & 1538 & 1.7 \\
\hline Southern Europe & 1001 & 1.2 & 1040 & 1.2 & 926 & 1.1 & 927 & 1.0 & 901 & 1.0 \\
\hline Western \& Northern Europe & 614 & 0.7 & 660 & 0.8 & 649 & 0.8 & 688 & 0.8 & 725 & 0.8 \\
\hline $\begin{array}{l}\text { Eastern Europe, Russia, Central Asian \& } \\
\text { Baltic States }\end{array}$ & 458 & 0.5 & 486 & 0.6 & 518 & 0.6 & 578 & 0.6 & 564 & 0.6 \\
\hline Middle East \& Africa & 3653 & 4.3 & 3879 & 4.6 & 3876 & 4.6 & 4080 & 4.6 & 4216 & 4.6 \\
\hline South East Asia & 4557 & 5.4 & 4673 & 5.5 & 4445 & 5.3 & 4571 & 5.1 & 4698 & 5.1 \\
\hline North East Asia & 2962 & 3.5 & 2819 & 3.3 & 2884 & 3.4 & 3117 & 3.5 & 3143 & 3.4 \\
\hline Southern Asia & 1716 & 2.0 & 1746 & 2.1 & 1930 & 2.3 & 2396 & 2.7 & 2759 & 3.0 \\
\hline Other-not stated & 76 & 0.1 & 170 & 0.2 & 180 & 0.2 & 218 & 0.2 & 491 & 0.5 \\
\hline TOTAL & 84587 & 100.0 & 85032 & 100.0 & 84288 & 100.0 & 89140 & 100.0 & 91315 & 100.0 \\
\hline \multicolumn{11}{|l|}{ Births } \\
\hline English-speaking & 68458 & 79.6 & 68459 & 79.2 & 67735 & 79.1 & 71558 & 79.0 & 72699 & 78.4 \\
\hline Central \& South America & 755 & 0.9 & 699 & 0.8 & 683 & 0.8 & 743 & 0.8 & 779 & 0.8 \\
\hline Melanesia, Micronesia \& Polynesia & 1555 & 1.8 & 1564 & 1.8 & 1600 & 1.9 & 1508 & 1.7 & 1564 & 1.7 \\
\hline Southern Europe & 1022 & 1.2 & 1064 & 1.2 & 939 & 1.1 & 945 & 1.0 & 920 & 1.0 \\
\hline Western \& Northern Europe & 627 & 0.7 & 668 & 0.8 & 663 & 0.8 & 700 & 0.8 & 737 & 0.8 \\
\hline $\begin{array}{l}\text { Eastern Europe, Russia, Central Asian \& } \\
\text { Baltic States }\end{array}$ & 468 & 0.5 & 494 & 0.6 & 525 & 0.6 & 593 & 0.7 & 573 & 0.6 \\
\hline Middle East \& Africa & 3711 & 4.3 & 3947 & 4.6 & 3941 & 4.6 & 4142 & 4.6 & 4287 & 4.6 \\
\hline South East Asia & 4595 & 5.3 & 4733 & 5.5 & 4488 & 5.2 & 4626 & 5.1 & 4746 & 5.1 \\
\hline North East Asia & 3000 & 3.5 & 2846 & 3.3 & 2910 & 3.4 & 3144 & 3.5 & 3172 & 3.4 \\
\hline Southern Asia & 1738 & 2.0 & 1766 & 2.0 & 1953 & 2.3 & 2428 & 2.7 & 2801 & 3.0 \\
\hline Other-not stated & 76 & 0.1 & 174 & 0.2 & 189 & 0.2 & 223 & 0.2 & 503 & 0.5 \\
\hline TOTAL & 86005 & 100.0 & 86414 & 100.0 & 85626 & 100.0 & 90610 & 100.0 & 92781 & 100.0 \\
\hline
\end{tabular}




\section{Maternal age}

Births to teenage mothers were more common among mothers born in English-speaking countries than non-English speaking countries (Table 86, Figure 4), while the largest proportions of mothers aged 35 years and over were among mothers born in Western and Northern Europe (38.9 per cent) and North East Asia (31.7 per cent).

\section{TABLE 86}

MATERNAL AGE BY COUNTRY OF BIRTH GROUP, NSW 2006

Country of birth group

\begin{tabular}{|c|c|c|c|c|c|c|c|c|c|c|}
\hline & \multicolumn{10}{|c|}{ iviatemial age (yeais) } \\
\hline & \multicolumn{2}{|c|}{$12-19$} & \multicolumn{2}{|c|}{ 20-34 } & \multicolumn{2}{|c|}{$35+$} & \multicolumn{2}{|c|}{ Not stated } & \multicolumn{2}{|c|}{2006} \\
\hline & No. & $\%$ & No. & $\%$ & No. & $\%$ & No. & $\%$ & No. & $\%$ \\
\hline English-speaking & 3231 & 4.5 & 53312 & 74.5 & 14924 & 20.9 & 48 & 0.1 & 71515 & 100.0 \\
\hline Central \& South America & 9 & 1.2 & 516 & 67.5 & 240 & 31.4 & 0 & 0.0 & 765 & 100.0 \\
\hline Melanesia, Micronesia \& Polynesia & 24 & 1.6 & 1146 & 74.5 & 368 & 23.9 & 0 & 0.0 & 1538 & 100.0 \\
\hline Southern Europe & 7 & 0.8 & 625 & 69.4 & 269 & 29.9 & 0 & 0.0 & 901 & 100.0 \\
\hline Western \& Northern Europe & 3 & 0.4 & 439 & 60.6 & 282 & 38.9 & 1 & 0.1 & 725 & 100.0 \\
\hline $\begin{array}{l}\text { Eastern Europe, Russia, Central Asian } \\
\text { \& Baltic States }\end{array}$ & 1 & 0.2 & 456 & 80.9 & 107 & 19.0 & 0 & 0.0 & 564 & 100.0 \\
\hline Middle East \& Africa & 107 & 2.5 & 3210 & 76.1 & 898 & 21.3 & 1 & 0.0 & 4216 & 100.0 \\
\hline South East Asia & 42 & 0.9 & 3502 & 74.5 & 1153 & 24.5 & 1 & 0.0 & 4698 & 100.0 \\
\hline North East Asia & 13 & 0.4 & 2134 & 67.9 & 995 & 31.7 & 1 & 0.0 & 3143 & 100.0 \\
\hline Southern Asia & 23 & 0.8 & 2355 & 85.4 & 381 & 13.8 & 0 & 0.0 & 2759 & 100.0 \\
\hline Other-not stated & 11 & 2.2 & 329 & 67.0 & 145 & 29.5 & 6 & 1.2 & 491 & 100.0 \\
\hline TOTAL & 3471 & 3.8 & 68024 & 74.5 & 19762 & 21.6 & 58 & 0.1 & 91315 & 100.0 \\
\hline
\end{tabular}

\section{FIGURE 4}

\section{MATERNAL AGE BY COUNTRY OF BIRTH GROUP, NSW 2002-2006}

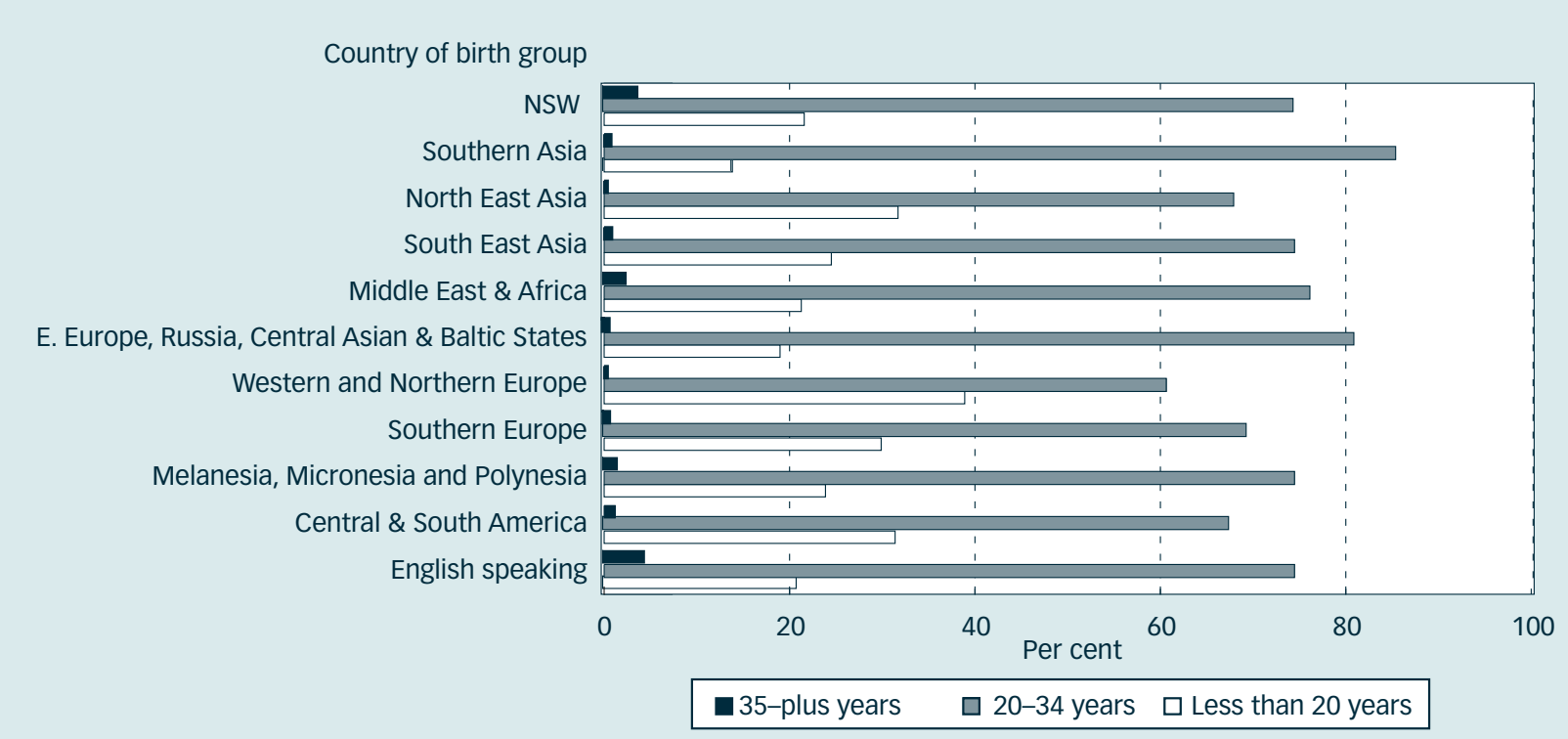

Source: NSW Midwives Data Collection (HOIST). Centre for Epidemiology and Research. NSW Department of Health. 


\section{Health area of residence}

In 2006, the proportion of mothers born in non-English speaking countries was highest in the Sydney South West Area (37.7 per cent), followed by the Sydney West Area (31.0 per cent). Five per cent of mothers were born in South East Asian countries, 50.3 per cent of whom were resident in the Sydney South West Area. Almost 5 per cent of mothers were born in Middle Eastern or African countries and 80.9 per cent of these mothers were resident in the Sydney South West or Sydney West Areas. A further 3.4 per cent of mothers were born in North East Asian countries and 3.0 per cent in Southern Asian countries, with the majority living in metropolitan areas (Table 87).

\section{TABLE 87}

\section{HEALTH AREA OF RESIDENCE BY MATERNAL COUNTRY OF BIRTH GROUP, NSW 2006\#}

\begin{tabular}{|c|c|c|c|c|c|c|c|c|c|c|c|c|c|c|c|c|c|c|c|c|c|c|c|}
\hline \multirow{3}{*}{$\begin{array}{l}\text { Health } \\
\text { Area }\end{array}$} & \multicolumn{23}{|c|}{ Country of birth group } \\
\hline & $\begin{array}{l}\text { English } \\
\text { speaking }\end{array}$ & \multicolumn{2}{|c|}{$\begin{array}{l}\text { Central \& } \\
\text { South } \\
\text { America }\end{array}$} & \multicolumn{2}{|c|}{$\begin{array}{c}\text { Melanesia, } \\
\text { Micronesia, } \\
\text { Polynesia }\end{array}$} & \multicolumn{2}{|c|}{$\begin{array}{l}\text { Southern } \\
\text { Europe }\end{array}$} & \multicolumn{2}{|c|}{$\begin{array}{l}\text { Western \& } \\
\text { Northern } \\
\text { Europe }\end{array}$} & \multicolumn{2}{|c|}{$\begin{array}{c}\text { Eastern } \\
\text { Europe, } \\
\text { Russia, } \\
\text { Central } \\
\text { Asian \& } \\
\text { Baltic } \\
\text { States }\end{array}$} & \multicolumn{2}{|c|}{$\begin{array}{l}\text { Middle } \\
\text { East \& } \\
\text { Africa }\end{array}$} & \multicolumn{2}{|c|}{$\begin{array}{l}\text { South East I } \\
\text { Asia }\end{array}$} & \multicolumn{2}{|c|}{$\begin{array}{l}\text { North East } \\
\text { Asia }\end{array}$} & \multicolumn{2}{|c|}{$\begin{array}{l}\text { Southern } \\
\text { Asia }\end{array}$} & \multicolumn{2}{|c|}{$\begin{array}{l}\text { Other- } \\
\text { not } \\
\text { stated }\end{array}$} & \multicolumn{2}{|c|}{ TOTAL } \\
\hline & No. $\%$ & No. & $\%$ & No. & $\%$ & No. & $\%$ & No. & $\%$ & No. & $\%$ & No. & $\%$ & No. & $\%$ & No. & $\%$ & No. & $\%$ & No. & $\%$ & No. & $\%$ \\
\hline $\begin{array}{l}\text { Sydney South } \\
\text { West }\end{array}$ & $12614 \quad 61.8$ & 292 & 1.4 & 673 & 3.3 & 418 & 2.0 & 141 & 0.7 & 140 & 0.7 & 2030 & 9.9 & 2363 & 11.6 & 897 & 4.4 & 748 & 3.7 & 110 & 0.5 & 20426 & 100.0 \\
\hline $\begin{array}{l}\text { South Eastern } \\
\text { Sydney \& } \\
\text { Illawarra }\end{array}$ & 1196479.7 & 175 & 1.2 & 126 & 0.8 & 219 & 1.5 & 180 & 1.2 & 149 & 1.0 & 456 & 3.0 & 645 & 4.3 & 650 & 4.3 & 266 & 1.8 & 190 & 1.3 & 15020 & 100.0 \\
\hline Sydney West & 1183368.8 & 115 & 0.7 & 533 & 3.1 & 138 & 0.8 & 74 & 0.4 & 114 & 0.7 & 1379 & 8.0 & 969 & 5.6 & 682 & 4.0 & 1323 & 7.7 & 50 & 0.3 & 17210 & 100.0 \\
\hline $\begin{array}{l}\text { Northern } \\
\text { Sydney \& } \\
\text { Central Coast }\end{array}$ & 1163982.3 & 137 & 1.0 & 94 & 0.7 & 92 & 0.7 & 210 & 1.5 & 126 & 0.9 & 226 & 1.6 & 470 & 3.3 & 774 & 5.5 & 321 & 2.3 & 50 & 0.4 & 14139 & 100.0 \\
\hline $\begin{array}{l}\text { Hunter \& New } \\
\text { England }\end{array}$ & 1015095.9 & 17 & 0.2 & 48 & 0.5 & 20 & 0.2 & 44 & 0.4 & 16 & 0.2 & 49 & 0.5 & 115 & 1.1 & 61 & 0.6 & 40 & 0.4 & 19 & 0.2 & 10579 & 100.0 \\
\hline North Coast & 489195.9 & 11 & 0.2 & 15 & 0.3 & - & 0.1 & 37 & 0.7 & 5 & 0.1 & 28 & 0.5 & 54 & 1.1 & 27 & 0.5 & 14 & 0.3 & - & 0.3 & 5100 & 100.0 \\
\hline $\begin{array}{l}\text { Greater } \\
\text { Southern }\end{array}$ & 388495.7 & 6 & 0.1 & 31 & 0.8 & - & 0.1 & 19 & 0.5 & - & 0.1 & 23 & 0.6 & 31 & 0.8 & 17 & 0.4 & 27 & 0.7 & 14 & 0.3 & 4059 & 100.0 \\
\hline $\begin{array}{l}\text { Greater } \\
\text { Western }\end{array}$ & 391196.5 & 5 & 0.1 & 9 & 0.2 & - & 0.1 & 11 & 0.3 & 7 & 0.2 & 16 & 0.4 & 29 & 0.7 & 11 & 0.3 & 12 & 0.3 & - & 0.9 & 4052 & 100.0 \\
\hline $\begin{array}{l}\text { Other-not } \\
\text { stated }\end{array}$ & 62986.2 & 7 & 1.0 & 9 & 1.2 & 3 & 0.4 & 9 & 1.2 & 4 & 0.5 & 9 & 1.2 & 22 & 3.0 & 24 & 3.3 & 8 & 1.1 & 6 & 0.8 & 730 & 100.0 \\
\hline TOTAL & 7151578.3 & 765 & 0.8 & 1538 & 1.7 & 901 & 1.0 & 725 & 0.8 & 564 & 0.6 & 4216 & 4.6 & 4698 & 5.1 & 3143 & 3.4 & 2759 & 3.0 & 491 & 0.5 & 91315 & 100.0 \\
\hline
\end{tabular}




\section{Duration of pregnancy at first antenatal visit}

In 2006, 87.5 per cent of all mothers commenced antenatal care before 20 weeks gestation. There was some variation between country of birth groups, with 89.6 per cent of mothers born in English speaking countries commencing antenatal care before 20 weeks gestation, compared with 64.9 per cent of mothers born in Melanesia, Micronesia, and Polynesia, and 72.8 per cent of mothers born in the Middle East and Africa (Table 88).

\begin{tabular}{|c|c|c|c|c|c|c|c|c|}
\hline \multirow[t]{3}{*}{ Country of birth group } & \multicolumn{8}{|c|}{ Duration of pregnancy at first antenatal visit (weeks) } \\
\hline & \multicolumn{2}{|c|}{ 0-19 } & \multicolumn{2}{|c|}{ 20-plus } & \multicolumn{2}{|c|}{ Not stated } & \multicolumn{2}{|c|}{ TOTAL } \\
\hline & No. & $\%$ & No. & $\%$ & No. & $\%$ & No. & $\%$ \\
\hline English-speaking & 64042 & 89.6 & 6523 & 9.1 & 950 & 1.3 & 71515 & 100.0 \\
\hline Central \& South America & 673 & 88.0 & 86 & 11.2 & 6 & 0.8 & 765 & 100.0 \\
\hline Melanesia, Micronesia \& Polynesia & 998 & 64.9 & 517 & 33.6 & 23 & 1.5 & 1538 & 100.0 \\
\hline Southern Europe & 801 & 88.9 & 89 & 9.9 & 11 & 1.2 & 901 & 100.0 \\
\hline Western \& Northern Europe & 649 & 89.5 & 62 & 8.6 & 14 & 1.9 & 725 & 100.0 \\
\hline $\begin{array}{l}\text { Eastern Europe, Russia, Central Asian \& } \\
\text { Baltic States }\end{array}$ & 502 & 89.0 & 57 & 10.1 & 5 & 0.9 & 564 & 100.0 \\
\hline Middle East \& Africa & 3068 & 72.8 & 1099 & 26.1 & 49 & 1.2 & 4216 & 100.0 \\
\hline South East Asia & 3843 & 81.8 & 788 & 16.8 & 67 & 1.4 & 4698 & 100.0 \\
\hline North East Asia & 2611 & 83.1 & 501 & 15.9 & 31 & 1.0 & 3143 & 100.0 \\
\hline Southern Asia & 2288 & 82.9 & 453 & 16.4 & 18 & 0.7 & 2759 & 100.0 \\
\hline Other-not stated & 399 & 81.3 & 43 & 8.8 & 49 & 10.0 & 491 & 100.0 \\
\hline TOTAL & 79874 & 87.5 & 10218 & 11.2 & 1223 & 1.3 & 91315 & 100.0 \\
\hline
\end{tabular}

Source: NSW Midwives Data Collection, Centre for Epidemiology and Research, NSW Department of Health.

\section{Smoking in pregnancy}

In 2006, smoking at any time during pregnancy was more common among mothers born in English-speaking countries than mothers born in non-English-speaking countries (Table 89). About one in 6 mothers born in English-speaking countries smoked at some time during pregnancy, compared to fewer than one in 9 mothers born in other country of birth groups.
Smoking in the second half of pregnancy poses the greatest risk to the health of both mother and baby. Four per cent of mothers who smoked during pregnancy quit before the second half of pregnancy. Of mothers who did smoke in the second half of pregnancy, mothers born in Englishspeaking countries were more likely to smoke more than 10 cigarettes per day compared to mothers born in other country of birth groups (Table 90).

\section{TABLE 89}

SMOKING IN PREGNANCY BY COUNTRY OF BIRTH GROUP, NSW 2006

Country of birth group

Smoking in pregnancy

\begin{tabular}{lrrrrrrrr} 
& \multicolumn{2}{c}{ No } & \multicolumn{3}{c}{ Yes } & \multicolumn{2}{c}{ Not stated } & TOTAL \\
& No. & $\%$ & No. & $\%$ & No. & $\%$ & No. & $\%$ \\
\hline English-speaking & 58825 & 82.3 & 11618 & 16.2 & 1072 & 1.5 & 71515 & 100.0 \\
Central \& South America & 714 & 93.3 & 29 & 3.8 & 22 & 2.9 & 765 & 100.0 \\
Melanesia, Micronesia \& Polynesia & 1365 & 88.8 & 135 & 8.8 & 38 & 2.5 & 1538 & 100.0 \\
Southern Europe & 799 & 88.7 & 72 & 8.0 & 30 & 3.3 & 901 & 100.0 \\
Western \& Northern Europe & 671 & 92.6 & 36 & 5.0 & 18 & 2.5 & 725 & 100.0 \\
Eastern Europe, Russia, Central Asian \& & 532 & 94.3 & 15 & 2.7 & 17 & 3.0 & 564 & 100.0 \\
Baltic States & & & & & & & \\
Middle East \& Africa & 3933 & 93.3 & 207 & 4.9 & 76 & 1.8 & 4216 & 100.0 \\
South East Asia & 4470 & 95.1 & 101 & 2.1 & 127 & 2.7 & 4698 & 100.0 \\
North East Asia & 2993 & 95.2 & 40 & 1.3 & 110 & 3.5 & 3143 & 100.0 \\
Southern Asia & 2675 & 97.0 & 11 & 0.4 & 73 & 2.6 & 2759 & 100.0 \\
Other-not stated & 438 & 89.2 & 43 & 8.8 & 10 & 2.0 & 491 & 100.0 \\
TOTAL & 77415 & 84.8 & 12307 & 13.5 & 1593 & 1.7 & 91315 & 100.0 \\
\hline Source: NSW Midwives Data Collection, Centre for Epidemiology and Research, NSW Department of Health. & & \\
\hline
\end{tabular}




\begin{tabular}{|c|c|c|c|c|c|c|c|c|c|c|c|c|}
\hline \multirow[t]{3}{*}{ Country of birth group } & \multicolumn{12}{|c|}{ Cigarettes per day in the second half of pregnancy } \\
\hline & \multicolumn{2}{|c|}{ None } & \multicolumn{2}{|c|}{ 1-10 per day } & \multicolumn{2}{|c|}{$\begin{array}{l}\text { More than } 10 \\
\text { per day }\end{array}$} & \multicolumn{2}{|c|}{$\begin{array}{l}\text { Smoked amount } \\
\text { not stated }\end{array}$} & \multicolumn{2}{|c|}{ Not stated } & \multicolumn{2}{|c|}{ TOTAL } \\
\hline & No. & $\%$ & No. & $\%$ & No. & $\%$ & No. & $\%$ & No. & $\%$ & No. & $\%$ \\
\hline English-speaking & 480 & 4.1 & 5819 & 50.1 & 4826 & 41.5 & 419 & 3.6 & 74 & 0.6 & 11618 & 100.0 \\
\hline Central \& South America & 3 & 10.3 & 19 & 65.5 & 5 & 17.2 & 2 & 6.9 & 0 & 0.0 & 29 & 100.0 \\
\hline $\begin{array}{l}\text { Melanesia, Micronesia \& } \\
\text { Polynesia }\end{array}$ & 1 & 0.7 & 95 & 70.4 & 36 & 26.7 & 3 & 2.2 & 0 & 0.0 & 135 & 100.0 \\
\hline Southern Europe & 3 & 4.2 & 45 & 62.5 & 20 & 27.8 & 4 & 5.6 & 0 & 0.0 & 72 & 100.0 \\
\hline Western \& Northern Europe & 1 & 2.8 & 22 & 61.1 & 10 & 27.8 & 3 & 8.3 & 0 & 0.0 & 36 & 100.0 \\
\hline $\begin{array}{l}\text { Eastern Europe, Russia, Central } \\
\text { Asian \& Baltic States }\end{array}$ & 0 & 0.0 & 10 & 66.7 & 5 & 33.3 & 0 & 0.0 & 0 & 0.0 & 15 & 100.0 \\
\hline Middle East \& Africa & 7 & 3.4 & 114 & 55.1 & 77 & 37.2 & 9 & 4.3 & 0 & 0.0 & 207 & 100.0 \\
\hline South East Asia & 10 & 9.9 & 64 & 63.4 & 20 & 19.8 & 5 & 5.0 & 2 & 2.0 & 101 & 100.0 \\
\hline North East Asia & 5 & 12.5 & 28 & 70.0 & 1 & 2.5 & 5 & 12.5 & 1 & 2.5 & 40 & 100.0 \\
\hline Southern Asia & 2 & 18.2 & 6 & 54.5 & 2 & 18.2 & 1 & 9.1 & 0 & 0.0 & 11 & 100.0 \\
\hline Other-not stated & 2 & 4.7 & 21 & 48.8 & 14 & 32.6 & 4 & 9.3 & 2 & 4.7 & 43 & 100.0 \\
\hline TOTAL & 514 & 4.2 & 6243 & 50.7 & 5016 & 40.8 & 455 & 3.7 & 79 & 0.6 & 12307 & 100.0 \\
\hline
\end{tabular}

\section{Medical conditions and obstetric complications}

In 2006, 1.2 per cent of mothers born in Melanesia, Micronesia, and Polynesia were reported to have diabetes mellitus - twice the rate for all mothers in NSW, though the number of mothers is small (Table 91). The rates of gestational diabetes in mothers born in Asian countries were over twice the rate for all mothers in NSW.
Overall, 0.9 per cent of mothers were reported to have essential hypertension, and 3.5 per cent were reported to have pre-eclampsia.

\section{TABLE 91}

MATERNAL MEDICAL CONDITIONS AND OBSTETRIC COMPLICATIONS BY COUNTRY OF BIRTH GROUP, NSW 2006 Country of birth group

\begin{tabular}{|c|c|c|c|c|c|c|c|c|c|c|}
\hline \multirow[t]{2}{*}{ 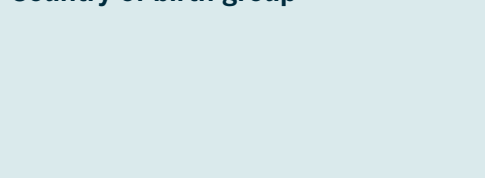 } & \multicolumn{2}{|c|}{$\begin{array}{l}\text { Diabetes } \\
\text { mellitus }\end{array}$} & \multicolumn{2}{|c|}{$\begin{array}{l}\text { Gestational } \\
\text { diabetes }\end{array}$} & \multicolumn{2}{|c|}{$\begin{array}{c}\text { Essential } \\
\text { hypertension }\end{array}$} & \multicolumn{2}{|c|}{$\begin{array}{l}\text { Pregnancy- } \\
\text { induced } \\
\text { hypertension }\end{array}$} & \multicolumn{2}{|c|}{ TOTAL } \\
\hline & No. & $\%$ & No. & $\%$ & No. & $\%$ & No. & $\%$ & No. & $\%$ \\
\hline English-speaking & 428 & 0.6 & 2527 & 3.5 & 692 & 1.0 & 3716 & 5.2 & 71515 & 100.0 \\
\hline Central \& South America & 4 & 0.5 & 46 & 6.0 & 7 & 0.9 & 39 & 5.1 & 765 & 100.0 \\
\hline Melanesia, Micronesia \& Polynesia & 18 & 1.2 & 175 & 11.4 & 6 & 0.4 & 82 & 5.3 & 1538 & 100.0 \\
\hline Southern Europe & 4 & 0.4 & 53 & 5.9 & 5 & 0.6 & 29 & 3.2 & 901 & 100.0 \\
\hline Western \& Northern Europe & 3 & 0.4 & 29 & 4.0 & 5 & 0.7 & 29 & 4.0 & 725 & 100.0 \\
\hline $\begin{array}{l}\text { Eastern Europe, Russia, Central Asian \& } \\
\text { Baltic States }\end{array}$ & 1 & 0.2 & 19 & 3.4 & 1 & 0.2 & 25 & 4.4 & 564 & 100.0 \\
\hline Middle East \& Africa & 25 & 0.6 & 320 & 7.6 & 29 & 0.7 & 133 & 3.2 & 4216 & 100.0 \\
\hline South East Asia & 24 & 0.5 & 518 & 11.0 & 26 & 0.6 & 158 & 3.4 & 4698 & 100.0 \\
\hline North East Asia & 11 & 0.3 & 312 & 9.9 & 19 & 0.6 & 93 & 3.0 & 3143 & 100.0 \\
\hline Southern Asia & 21 & 0.8 & 355 & 12.9 & 11 & 0.4 & 111 & 4.0 & 2759 & 100.0 \\
\hline Other-not stated & 2 & 0.4 & 21 & 4.3 & 3 & 0.6 & 21 & 4.3 & 491 & 100.0 \\
\hline TOTAL\# & 541 & 0.6 & 4375 & 4.8 & 804 & 0.9 & 4436 & 4.9 & 91315 & 100.0 \\
\hline
\end{tabular}




\section{Labour and birth}

Mothers born in non-English-speaking countries were more likely to have a spontaneous onset of labour than mothers born in English speaking countries, and were less likely to be induced (Table 92).
Mothers born in the Middle East and Africa and Melanesia, Micronesia and Polynesia were more likely to have a normal vaginal birth than mothers in other country of birth groups (Table 93). The highest caesarean section rates were among mothers born in Southern Asia (33.4 per cent) and Central and South America (33.3 per cent).

\section{TABLE 92}

\section{LABOUR ONSET BY COUNTRY OF BIRTH GROUP, NSW 2006}

\begin{tabular}{|c|c|c|c|c|c|c|c|c|c|c|}
\hline \multirow[t]{3}{*}{ Country of birth group } & \multicolumn{10}{|c|}{ Onset of labour } \\
\hline & \multicolumn{2}{|c|}{ Spontaneous } & \multicolumn{2}{|c|}{ No labour } & \multicolumn{2}{|c|}{ Induced } & \multicolumn{2}{|c|}{ Not stated } & \multicolumn{2}{|c|}{ TOTAL } \\
\hline & No. & $\%$ & No. & $\%$ & No. & $\%$ & No. & $\%$ & No. & $\%$ \\
\hline English-speaking & 40467 & 56.6 & 12359 & 17.3 & 18634 & 26.1 & 55 & 0.1 & 71515 & 100.0 \\
\hline Central \& South America & 460 & 60.1 & 133 & 17.4 & 172 & 22.5 & 0 & 0.0 & 765 & 100.0 \\
\hline Melanesia, Micronesia \& Polynesia & 1017 & 66.1 & 207 & 13.5 & 314 & 20.4 & 0 & 0.0 & 1538 & 100.0 \\
\hline Southern Europe & 547 & 60.7 & 144 & 16.0 & 210 & 23.3 & 0 & 0.0 & 901 & 100.0 \\
\hline Western \& Northern Europe & 464 & 64.0 & 116 & 16.0 & 145 & 20.0 & 0 & 0.0 & 725 & 100.0 \\
\hline $\begin{array}{l}\text { Eastern Europe, Russia, Central Asian \& } \\
\text { Baltic States }\end{array}$ & 355 & 62.9 & 89 & 15.8 & 120 & 21.3 & 0 & 0.0 & 564 & 100.0 \\
\hline Middle East \& Africa & 2715 & 64.4 & 590 & 14.0 & 910 & 21.6 & 1 & 0.0 & 4216 & 100.0 \\
\hline South East Asia & 3252 & 69.2 & 741 & 15.8 & 701 & 14.9 & 4 & 0.1 & 4698 & 100.0 \\
\hline North East Asia & 2033 & 64.7 & 517 & 16.4 & 591 & 18.8 & 2 & 0.1 & 3143 & 100.0 \\
\hline Southern Asia & 1608 & 58.3 & 479 & 17.4 & 670 & 24.3 & 2 & 0.1 & 2759 & 100.0 \\
\hline Other-not stated & 252 & 51.3 & 141 & 28.7 & 92 & 18.7 & 6 & 1.2 & 491 & 100.0 \\
\hline TOTAL\# & 53170 & 58.2 & 15516 & 17.0 & 22559 & 24.7 & 70 & 0.1 & 91315 & 100.0 \\
\hline
\end{tabular}

\section{TABLE 93}

\section{TYPE OF BIRTH BY COUNTRY OF BIRTH GROUP, NSW 2006}

Health Area

Type of birth

\begin{tabular}{|c|c|c|c|c|c|c|c|c|c|c|c|c|c|c|c|c|}
\hline & \multicolumn{2}{|c|}{$\begin{array}{l}\text { Normal } \\
\text { vaginal }\end{array}$} & \multicolumn{2}{|c|}{ Forceps } & \multicolumn{2}{|c|}{$\begin{array}{l}\text { Vacuum } \\
\text { extraction }\end{array}$} & \multicolumn{2}{|c|}{$\begin{array}{l}\text { Vaginal } \\
\text { breech }\end{array}$} & \multicolumn{2}{|c|}{$\begin{array}{c}\text { Elective } \\
\text { caesarean } \\
\text { section }\end{array}$} & \multicolumn{2}{|c|}{$\begin{array}{l}\text { Emergency } \\
\text { caesarean } \\
\text { section\# }\end{array}$} & \multicolumn{2}{|c|}{$\begin{array}{c}\text { Not } \\
\text { stated }\end{array}$} & \multicolumn{2}{|c|}{ TOTAL } \\
\hline & No. & $\%$ & No. & $\%$ & No. & $\%$ & No. & $\%$ & No. & $\%$ & No. & $\%$ & No. & $\%$ & No. & $\%$ \\
\hline English-speaking & 43195 & 60.4 & 2235 & 3.1 & 4889 & 6.8 & 285 & 0.4 & 12352 & 17.3 & 8383 & 11.7 & 176 & 0.2 & 71515 & 100.0 \\
\hline $\begin{array}{l}\text { Central \& South } \\
\text { America }\end{array}$ & 423 & 55.3 & 31 & 4.1 & 50 & 6.5 & 4 & 0.5 & 133 & 17.4 & 122 & 15.9 & 2 & 0.3 & 765 & 100.0 \\
\hline $\begin{array}{l}\text { Melanesia, Micronesia } \\
\text { \& Polynesia }\end{array}$ & 1070 & 69.6 & 28 & 1.8 & 62 & 4.0 & 9 & 0.6 & 207 & 13.5 & 162 & 10.5 & 0 & 0.0 & 1538 & 100.0 \\
\hline Southern Europe & 565 & 62.7 & 30 & 3.3 & 64 & 7.1 & 5 & 0.6 & 144 & 16.0 & 91 & 10.1 & 2 & 0.2 & 901 & 100.0 \\
\hline $\begin{array}{l}\text { Western \& Northern } \\
\text { Europe }\end{array}$ & 434 & 59.9 & 26 & 3.6 & 64 & 8.8 & 3 & 0.4 & 116 & 16.0 & 77 & 10.6 & 5 & 0.7 & 725 & 100.0 \\
\hline $\begin{array}{l}\text { Eastern Europe, Russia, } \\
\text { Central Asian \& Baltic } \\
\text { States }\end{array}$ & 332 & 58.9 & 23 & 4.1 & 44 & 7.8 & 2 & 0.4 & 89 & 15.8 & 72 & 12.8 & 2 & 0.4 & 564 & 100.0 \\
\hline Middle East \& Africa & 2909 & 69.0 & 84 & 2.0 & 197 & 4.7 & 13 & 0.3 & 590 & 14.0 & 420 & 10.0 & 3 & 0.1 & 4216 & 100.0 \\
\hline South East Asia & 2748 & 58.5 & 157 & 3.3 & 409 & 8.7 & 27 & 0.6 & 741 & 15.8 & 607 & 12.9 & 9 & 0.2 & 4698 & 100.0 \\
\hline North East Asia & 1785 & 56.8 & 123 & 3.9 & 322 & 10.2 & 6 & 0.2 & 517 & 16.4 & 387 & 12.3 & 3 & 0.1 & 3143 & 100.0 \\
\hline Southern Asia & 1487 & 53.9 & 132 & 4.8 & 210 & 7.6 & 7 & 0.3 & 477 & 17.3 & 445 & 16.1 & 1 & 0.0 & 2759 & 100.0 \\
\hline Other-not stated & 239 & 48.7 & 16 & 3.3 & 21 & 4.3 & 5 & 1.0 & 141 & 28.7 & 61 & 12.4 & 8 & 1.6 & 491 & 100.0 \\
\hline TOTAL & 55187 & 60.4 & 2885 & 3.2 & 6332 & 6.9 & 366 & 0.4 & 15507 & 17.0 & 10827 & 11.9 & 211 & 0.2 & 91315 & 100.0 \\
\hline
\end{tabular}




\section{Birth weight}

The rate of low birth weight (less than 2,500 grams) in 2006 was 6.4 per cent in NSW. The highest rates of low birth weight were in babies of mothers born in Southern Asia
(9.8 per cent) and Melanesia, Micronesia and Polynesia (8.1 per cent) (Table 94). Babies of mothers born in North East Asia were least likely to be low birth weight.

\section{TABLE 94}

\section{BIRTH WEIGHT BY MATERNAL COUNTRY OF BIRTH GROUP, NSW 2006}

Country of birth group

\begin{tabular}{|c|c|c|c|c|c|c|c|c|}
\hline & \multicolumn{2}{|c|}{ Less than 2500} & \multicolumn{2}{|c|}{$2500+$} & \multicolumn{2}{|c|}{ Not stated } & \multicolumn{2}{|c|}{ TOTAL } \\
\hline & No. & $\%$ & No. & $\%$ & No. & $\%$ & No. & $\%$ \\
\hline English-speaking & 4598 & 6.3 & 68006 & 93.5 & 95 & 0.1 & 72699 & 100.0 \\
\hline Central \& South America & 40 & 5.1 & 738 & 94.7 & 1 & 0.1 & 779 & 100.0 \\
\hline Melanesia, Micronesia \& Polynesia & 126 & 8.1 & 1435 & 91.8 & 3 & 0.2 & 1564 & 100.0 \\
\hline Southern Europe & 51 & 5.5 & 865 & 94.0 & 4 & 0.4 & 920 & 100.0 \\
\hline Western \& Northern Europe & 37 & 5.0 & 700 & 95.0 & 0 & 0.0 & 737 & 100.0 \\
\hline $\begin{array}{l}\text { Eastern Europe, Russia, Central Asian \& } \\
\text { Baltic States }\end{array}$ & 34 & 5.9 & 539 & 94.1 & 0 & 0.0 & 573 & 100.0 \\
\hline Middle East \& Africa & 263 & 6.1 & 4016 & 93.7 & 8 & 0.2 & 4287 & 100.0 \\
\hline South East Asia & 314 & 6.6 & 4425 & 93.2 & 7 & 0.1 & 4746 & 100.0 \\
\hline North East Asia & 142 & 4.5 & 3027 & 95.4 & 3 & 0.1 & 3172 & 100.0 \\
\hline Southern Asia & 274 & 9.8 & 2525 & 90.1 & 2 & 0.1 & 2801 & 100.0 \\
\hline Other-not stated & 54 & 10.7 & 447 & 88.9 & 2 & 0.4 & 503 & 100.0 \\
\hline TOTAL & 5933 & 6.4 & 86723 & 93.5 & 125 & 0.1 & 92781 & 100.0 \\
\hline
\end{tabular}

\section{Gestational age}

The rate of prematurity (less than 37 weeks gestation) in 2006 was 7.4 per cent in NSW. The highest rates of prematurity were in babies of mothers born in Melanesia, Micronesia and
Polynesia (8.7 per cent) and Central and South America (8.0 per cent). Babies of mothers born in North East Asia were least likely to be premature (Table 95).

\section{TABLE 95}

GESTATIONAL AGE BY MATERNAL COUNTRY OF BIRTH GROUP, NSW 2006

Country of birth group

Gestational age (weeks)

\begin{tabular}{|c|c|c|c|c|c|c|c|c|}
\hline & \multicolumn{2}{|c|}{ Less than 37} & \multicolumn{2}{|c|}{$37+$} & \multicolumn{2}{|c|}{ Not stated } & \multicolumn{2}{|c|}{ TOTAL } \\
\hline & No. & $\%$ & No. & $\%$ & No. & $\%$ & No. & $\%$ \\
\hline English-speaking & 5445 & 7.5 & 67240 & 92.5 & 14 & 0.0 & 72699 & 100.0 \\
\hline Central \& South America & 62 & 8.0 & 717 & 92.0 & 0 & 0.0 & 779 & 100.0 \\
\hline Melanesia, Micronesia \& Polynesia & 136 & 8.7 & 1427 & 91.2 & 1 & 0.1 & 1564 & 100.0 \\
\hline Southern Europe & 58 & 6.3 & 862 & 93.7 & 0 & 0.0 & 920 & 100.0 \\
\hline Western \& Northern Europe & 45 & 6.1 & 692 & 93.9 & 0 & 0.0 & 737 & 100.0 \\
\hline $\begin{array}{l}\text { Eastern Europe, Russia, Central Asian \& } \\
\text { Baltic States }\end{array}$ & 35 & 6.1 & 538 & 93.9 & 0 & 0.0 & 573 & 100.0 \\
\hline Middle East \& Africa & 290 & 6.8 & 3996 & 93.2 & 1 & 0.0 & 4287 & 100.0 \\
\hline South East Asia & 356 & 7.5 & 4390 & 92.5 & 0 & 0.0 & 4746 & 100.0 \\
\hline North East Asia & 159 & 5.0 & 3013 & 95.0 & 0 & 0.0 & 3172 & 100.0 \\
\hline Southern Asia & 204 & 7.3 & 2597 & 92.7 & 0 & 0.0 & 2801 & 100.0 \\
\hline Other-not stated & 49 & 9.7 & 451 & 89.7 & 3 & 0.6 & 503 & 100.0 \\
\hline TOTAL & 6839 & 7.4 & 85923 & 92.6 & 19 & 0.0 & 92781 & 100.0 \\
\hline
\end{tabular}




\section{Apgar score}

In 2006, 2.0 per cent of all babies (including stillborn babies) had an Apgar score of 7 or less at 5 minutes and 1.1 per cent had a score of less than 4 (Table 96). The rate of
Apgar scores of less than 7 was highest among babies of mothers born in the Middle East and Africa (2.5 per cent) and Melanesia, Micronesia and Polynesia (2.4 per cent).

\section{TABLE 96}

\section{BIRTHS BY COUNTRY OF BIRTH GROUP AND APGAR SCORE AT FIVE MINUTES, NSW 2006 Country of birth group Apgar Score}

\begin{tabular}{|c|c|c|c|c|c|c|c|c|c|c|}
\hline & \multicolumn{2}{|c|}{$0-4$} & \multicolumn{2}{|c|}{$5-6$} & \multicolumn{2}{|c|}{$7+$} & \multicolumn{2}{|c|}{ Not stated } & \multicolumn{2}{|c|}{ TOTAL } \\
\hline & No. & $\%$ & No. & $\%$ & No. & $\%$ & No. & $\%$ & No. & $\%$ \\
\hline English-speaking & 770 & 1.1 & 714 & 1.0 & 71017 & 97.7 & 198 & 0.3 & 72699 & 100.0 \\
\hline Central \& South America & 8 & 1.0 & 5 & 0.6 & 765 & 98.2 & 1 & 0.1 & 779 & 100.0 \\
\hline Melanesia, Micronesia \& Polynesia & 19 & 1.2 & 19 & 1.2 & 1520 & 97.2 & 6 & 0.4 & 1564 & 100.0 \\
\hline Southern Europe & 9 & 1.0 & 5 & 0.5 & 903 & 98.2 & 3 & 0.3 & 920 & 100.0 \\
\hline Western \& Northern Europe & 7 & 0.9 & 4 & 0.5 & 724 & 98.2 & 2 & 0.3 & 737 & 100.0 \\
\hline $\begin{array}{l}\text { Eastern Europe, Russia, Central Asian \& } \\
\text { Baltic States }\end{array}$ & 7 & 1.2 & 4 & 0.7 & 562 & 98.1 & 0 & 0.0 & 573 & 100.0 \\
\hline Middle East \& Africa & 70 & 1.6 & 36 & 0.8 & 4167 & 97.2 & 14 & 0.3 & 4287 & 100.0 \\
\hline South East Asia & 62 & 1.3 & 47 & 1.0 & 4629 & 97.5 & 8 & 0.2 & 4746 & 100.0 \\
\hline North East Asia & 23 & 0.7 & 14 & 0.4 & 3132 & 98.7 & 3 & 0.1 & 3172 & 100.0 \\
\hline Southern Asia & 24 & 0.9 & 35 & 1.2 & 2740 & 97.8 & 2 & 0.1 & 2801 & 100.0 \\
\hline Other-not stated & 8 & 1.6 & 7 & 1.4 & 486 & 96.6 & 2 & 0.4 & 503 & 100.0 \\
\hline TOTAL\# & 1007 & 1.1 & 890 & 1.0 & 90645 & 97.7 & 239 & 0.3 & 92781 & 100.0 \\
\hline
\end{tabular}

\section{Perinatal outcomes}

In 2006, 99 per cent of babies born in NSW and reported to the MDC were born alive and survived until discharge from the hospital of birth (Table 97). The majority of perinatal deaths occurred among babies of mothers born in English-speaking countries $(n=622,75.8$ per cent). There were a further 58 deaths among babies of mothers born in the Middle East and Africa and 50 deaths among babies of mothers born in South East Asian countries - comprising 7.1 and 6.1 per cent respectively of all perinatal deaths reported to the MDC.

\section{TABLE 97}

PERINATAL OUTCOMES BY HEALTH AREA OF RESIDENCE, NSW 2006

Country of birth Perinatal outcome

\begin{tabular}{|c|c|c|c|c|c|c|c|c|c|c|c|c|c|}
\hline \multirow[t]{2}{*}{ Country of birth } & & $\begin{array}{l}\text { born } \\
\text { iving }\end{array}$ & & irth & & $\begin{array}{l}\text { 1atal } \\
\text { atal } \\
\text { eath }\end{array}$ & $\begin{array}{l}\text { Death } \\
\text { not }\end{array}$ & $\begin{array}{l}\text { age } \\
\text { ted }\end{array}$ & Not & ted & Tota & births & $\begin{array}{c}\text { Perinatal } \\
\text { mortality / } \\
1000 \\
\text { births }\end{array}$ \\
\hline & No. & $\%$ & No. & $\%$ & No. & $\%$ & No. & $\%$ & No. & $\%$ & No. & $\%$ & No. \\
\hline English-speaking & 71987 & 99.0 & 445 & 0.6 & 177 & 0.2 & 6 & 0.0 & 84 & 0.1 & 72699 & 100.0 & 8.6 \\
\hline Central \& South America & 772 & 99.1 & 5 & 0.6 & 2 & 0.3 & 0 & 0.0 & 0 & 0.0 & 779 & 100.0 & 9.0 \\
\hline $\begin{array}{l}\text { Melanesia, Micronesia } \\
\& \text { Polynesia }\end{array}$ & 1544 & 98.7 & 13 & 0.8 & 4 & 0.3 & 0 & 0.0 & 3 & 0.2 & 1564 & 100.0 & 10.9 \\
\hline Southern Europe & 911 & 99.0 & 4 & 0.4 & 4 & 0.4 & 0 & 0.0 & 1 & 0.1 & 920 & 100.0 & 8.7 \\
\hline $\begin{array}{l}\text { Western \& Northern } \\
\text { Europe }\end{array}$ & 731 & 99.2 & 5 & 0.7 & 0 & 0.0 & 0 & 0.0 & 1 & 0.1 & 737 & 100.0 & 6.8 \\
\hline $\begin{array}{l}\text { Eastern Europe, Russia, } \\
\text { Central Asian \& Baltic } \\
\text { States }\end{array}$ & 566 & 98.8 & 6 & 1.0 & 1 & 0.2 & 0 & 0.0 & 0 & 0.0 & 573 & 100.0 & 12.2 \\
\hline Middle East \& Africa & 4224 & 98.5 & 40 & 0.9 & 18 & 0.4 & 0 & 0.0 & 5 & 0.1 & 4287 & 100.0 & 13.5 \\
\hline South East Asia & 4686 & 98.7 & 39 & 0.8 & 11 & 0.2 & 0 & 0.0 & 10 & 0.2 & 4746 & 100.0 & 10.5 \\
\hline North East Asia & 3152 & 99.4 & 12 & 0.4 & 6 & 0.2 & 0 & 0.0 & 2 & 0.1 & 3172 & 100.0 & 5.7 \\
\hline Southern Asia & 2774 & 99.0 & 18 & 0.6 & 4 & 0.1 & 0 & 0.0 & 5 & 0.2 & 2801 & 100.0 & 7.9 \\
\hline Other - not stated & 731 & 98.4 & 5 & 1.0 & 2 & 0.4 & 0 & 0.0 & 3 & 0.6 & 503 & 100.0 & - \\
\hline TOTAL & 91840 & 99.0 & 592 & 0.6 & 229 & 0.2 & 6 & 0.0 & 114 & 0.1 & 92781 & 100.0 & 8.8 \\
\hline
\end{tabular}

Source: NSW Midwives Data Collection (HOIST). Centre for Epidemiology and Research, NSW Department of Health.

\# Perinatal deaths include deaths reported to the MDC only. As the MDC form is completed at discharge or transfer of the baby; deaths occurring after this time may not be reported to the MDC. 